\title{
An introduction to the two-dimensional Schrödinger equation with nonlinear point interactions
}

\author{
R. Carlone ${ }^{1}$, M. Correggi ${ }^{2}$, L. Tentarelli ${ }^{2}$ \\ ${ }^{1}$ Università "Federico II" di Napoli, Dipartimento di Matematica e Applicazioni "R. Caccioppoli” \\ MSA, via Cinthia, I-80126, Napoli, Italy \\ 2 “Sapienza" Università di Roma, Dipartimento di Matematica P.le Aldo Moro, 5, 00185, Roma, Italy \\ raffaele.carlone@unina.it, michele.correggi@gmail.com, tentarelli@mat.uniroma1.it
}

PACS 03.65.-w, 02.30.Rz

DOI 10.17586/2220-8054-2018-9-2-187-195

We present an introduction to the nonlinear Schrödinger equation (NLSE) with concentrated nonlinearities in $\mathbb{R}^{2}$. Precisely, taking a cue from the linear problem, we sketch the main challenges and the typical difficulties that arise in the two-dimensional case, and mention some recent results obtained by the authors on local and global well-posedness.

Keywords: nonlinear Schrödinger equation, nonlinear delta interactions.

Received: 30 January 2018

Revised: 10 February 2018

\section{Introduction}

In the last twenty years the Schrödinger equation with point interactions has proven to be a very useful mathematical tool for modeling many interesting phenomena in several areas of theoretical physics: from foundations of quantum mechanics (e.g., [1-4]) to acoustics (e.g., [5]), from quantum field theory (e.g., [6]) to spectral theory (e.g., [7]). In addition, linear and nonlinear point interactions can be seen as singular perturbations not only of the standard Schrödinger (or heat) equation. They may also appear, for instance, in the study of the Dirac equation, a model which has recently attracted some renewed attention (see e.g., [8-10]).

Linear point interactions arise as a particular, but relevant, application of the more general theory of self-adjoint extension of symmetric operators; a theory that has gained new popularity in recent years also for the application to the study of evolution equations in non-standard domains, such as quantum graphs (see e.g., [11-26]) and quantum hybrids (see e.g., [27-31]).

The extension to nonlinear point interactions appeared first in [32] and its interest is driven by the possibility of investigating nonlinear problems in the context of solvable models (i.e., models with an explicit solution) such as, indeed, point interactions. In dimensions $\mathrm{d}=1,3$ these problems have been extensively analyzed and many results have been obtained, such as local and global well-posedness (see [32,33]), occurrence of blow-up solutions (see $[32,34]$ ), and approximation by standard NLSEs with concentrating potentials (the so-called point-like limit - see [35,36]). We also mention that in these dimensions also linear non-autonomous models have been widely studied, mainly in relation to complete ionization phoenomena (see e.g., [37-40]).

On the contrary, to the best of our knowledge, the two-dimensional problem has failed to be understood for years, even though some of the main technical difficulties arising in this case were known (see [7,8,41]). However, the problem has been finally solved by the authors in [42], where new features of Volterra integral operators with highly singular kernels have been established (see also [43]) in order to prove local and global well-posedness of the associated Cauchy problem.

In this communication, starting from linear point interactions in $\mathbb{R}^{2}$, we sketch some important points of the strategy used in [42] to discuss the issues of local and global well-posedness of the nonlinear model.

\section{Linear Point Interactions}

In this section, we give a brief overview on linear point interactions in $\mathbb{R}^{2}$ (for a more detailed, we refer to $[8])$.

As in $\mathbb{R}^{3}$ (and in contrast to what occurs in $\mathbb{R}$ ), in $\mathbb{R}^{2}$ the starting point is that of giving a precise meaning to the formal operator:

$$
H^{f}:=-\Delta+\sum_{i=1}^{N} \alpha_{i} \delta\left(\cdot-\mathbf{y}_{i}\right), \quad \mathbf{y}_{1}, \ldots, \mathbf{y}_{N} \in \mathbb{R}^{2}, \quad \alpha_{1}, \ldots, \alpha_{N} \in \mathbb{R} .
$$


Hence, we look for a suitable self-adjoint operator in $L^{2}\left(\mathbb{R}^{2}\right)$, which correctly represent the heuristic expression in (2.1). In particular, this operator has to act as the free Laplacian far from the points were the interactions occur.

In the following, we recall how to construct such an operator by means of the theory of self-adjoint extensions. For the sake of simplicity, we consider only the case of a single point interaction placed at $\mathbf{y} \in \mathbb{R}^{2}$ (for the generalization to the case of a finite number of point interactions see [8]).

\subsection{Definition and setting}

Let us introduce the following restriction of the Laplacian:

$$
H^{r}:=-\Delta, \quad \mathcal{D}\left(H^{r}\right):=C_{0}^{\infty}\left(\mathbb{R}^{2} \backslash\{\mathbf{y}\}\right) .
$$

This operator is symmetric and, furthermore, acts exactly as the standard Laplacian far from the interaction point. Hence, the strategy to find a suitable form for (2.1) is that of classifying all possible (non-trivial) self-adjoint extensions of $H^{r}$.

In fact, one can prove (see [8]) that all these extensions are given by a one-parameter family of operators $H_{\alpha, \mathbf{y}}$ with domain and action given by:

$$
\begin{gathered}
\mathcal{D}\left(H_{\alpha, \mathbf{y}}\right):=\left\{\psi \in L^{2}\left(\mathbb{R}^{2}\right) \mid \psi=\phi_{\lambda}+q G^{\lambda}(\cdot-\mathbf{y}), \quad \phi_{\lambda} \in H^{2}\left(\mathbb{R}^{2}\right), \quad q \in \mathbb{C},\right. \\
\left.\lim _{\mathbf{x} \rightarrow \mathbf{y}} \phi_{\lambda}(\mathbf{x})=\left(\alpha+\frac{1}{2 \pi} \log \sqrt{\lambda}+\frac{\gamma}{2 \pi}\right) q\right\}
\end{gathered}
$$

(for any $\lambda>0$ ) and

$$
\left(H_{\alpha, \mathbf{y}}+\lambda\right) \psi:=(-\Delta+\lambda) \phi_{\lambda}, \quad \forall \psi \in \mathcal{D}\left(H_{\alpha, \mathbf{y}}\right),
$$

where $\gamma$ is the Euler-Mascheroni constant and $G^{\lambda}$ is the Green's function of $-\Delta+\lambda$, namely $G^{\lambda}(\mathbf{x})=$ $\frac{1}{2 \pi} K_{0}(\sqrt{\lambda}|\mathbf{x}|)$, with $K_{0}(\sqrt{\lambda}|\cdot|)$ denoting the inverse (unitary) Fourier transform of $\left(|\mathbf{k}|^{2}+\lambda\right)^{-1}$, i.e., the modified Bessel function of second kind of order 0 (a.k.a. Macdonald function [49, Sect. 9.6]).

Remark 2.1. In the $3 \mathrm{~d}$ case, an analogous construction holds, but with a major difference: one can define an equivalent decomposition (up to modifying the integrability requirements at infinity) for $\lambda=0$. Here, on the contrary, although the operator domain is independent (as in 3d) of the parameter $\lambda>0$, the choice $\lambda=0$ is forbidden due to the infrared singularity of the $2 \mathrm{~d}$ Green's function (which in fact diverges when $\lambda \rightarrow 0$ ).

Remark 2.2. The parameter $\alpha$ introduced above is not the inverse scattering length. There is a relation between $\alpha$, defined above with the scattering length $\left(4 \pi \alpha^{\text {scatt }}\right)^{-1}$ as defined in [8], and it is the following:

$$
\alpha^{\text {scatt }}+\frac{\gamma}{2 \pi}-\frac{\log 2}{2 \pi}=\alpha .
$$

The quadratic form associated with $H_{\alpha, \mathbf{y}}$, in addition, is given by:

$$
\mathcal{F}_{\alpha, \mathbf{y}}(\psi):=\left\|\nabla \phi_{\lambda}\right\|_{L^{2}\left(\mathbb{R}^{2}\right)}^{2}+\lambda\left\|\phi_{\lambda}\right\|_{L^{2}\left(\mathbb{R}^{2}\right)}^{2}-\lambda\|\psi\|_{L^{2}\left(\mathbb{R}^{2}\right)}^{2}+\left(\alpha+\frac{1}{2 \pi} \log \frac{\sqrt{\lambda}}{2}+\frac{\gamma}{2 \pi}\right)|q|^{2},
$$

with form domain:

$$
V:=\left\{\psi \in L^{2}\left(\mathbb{R}^{2}\right) \mid \psi=\phi_{\lambda}+q G^{\lambda}(\cdot-\mathbf{y}), \phi_{\lambda} \in H^{1}\left(\mathbb{R}^{2}\right), \quad q \in \mathbb{C}\right\} .
$$

Such a quadratic form is not positive definite. As a consequence, one finds that a bound state occurs for any value of $\alpha$ (another major difference with the $3 \mathrm{~d}$ case, where the sign of the coupling constant $\alpha$ distinguishes between the existence/nonexistence of a bound state). More precisely:

$$
\sigma\left(H_{\alpha, \mathbf{y}}\right)=\left\{-4 e^{-4 \pi \alpha-2 \gamma}\right\} \cup[0, \infty),
$$

and thus two-dimensional point interactions can be said to be always attractive. 


\subsection{Dynamics}

As a consequence of the previous considerations (by means, for instance, of the Stone's theorem), it is well-known that, for any $\psi_{0} \in D\left(H_{\alpha, y}\right)$, the Cauchy problem:

$$
\left\{\begin{aligned}
\imath \frac{\partial \psi_{t}}{\partial t} & =H_{\alpha, \mathbf{y}} \psi_{t} \\
\psi_{t=0} & =\psi_{0}
\end{aligned}\right.
$$

is globally well-posed. Additionally, in this case, an expression for the propagator as an integral kernel is available (see again $[8,44]$ ). This means that the solution of the Cauchy problem can be given explicitly.

However, there exists an alternative description for the dynamics of (2.3), which has two main advantages. It makes the state description more similar to the physics intuition of what a point interaction is, and, especially, it is a suitable starting point for the generalization from linear problems to nonlinear problems, where no theory of self-adjoint operators is available.

This description is based on the following ansatz for the solutions:

$$
\psi_{t}(\mathbf{x})=\left(U_{0}(t) \psi_{0}\right)(\mathbf{x})+\frac{\imath}{2 \pi} \int_{0}^{t} \mathrm{~d} s U_{0}(t-s,|\mathbf{x}-\mathbf{y}|) q(s),
$$

where $U_{0}(t)$ is the integral kernel of the $2 \mathrm{~d}$ free Schrödinger propagator, i.e.,

$$
U_{0}(t ; \mathbf{x})=\frac{1}{2 \imath t} e^{-\frac{|\mathbf{x}|^{2}}{4 \imath t}}
$$

and $q(t)$ is a complex scalar function usually called charge. In this way, all the relevant information on the interaction is stored in fact in $q(t)$ and hence the dynamics of the problem is completely determined by the equation satisfied by $q(t)$, that is the so-called the charge equation.

Remark 2.3. At an intuitive level, (2.4) is simply the Duhamel formulation of (2.3) if one assumes that $q(t)$ represent the dynamics of the wave function at the interaction point.

Before justifying the previous ansatz on $\psi_{t}$ and deriving, at least formally, the charge equation, we need to introduce a technical tool (see also [42,43,45]). Recall that the Volterra functions (see, e.g., [46]) are defined as:

$$
\nu(t, \alpha):=\int_{0}^{\infty} \mathrm{d} s \frac{t^{\alpha+s}}{\Gamma(\alpha+s+1)},
$$

where $\Gamma(t):=\int_{0}^{\infty} \mathrm{d} x x^{t-1} e^{-x}$. In particular, we focus on the Volterra function of order -1 , i.e., $\nu(t,-1)=: \mathcal{I}(t)$. This function is finite (and analytic) for every $t>0$, whereas:

$$
\mathcal{I}(t) \sim \frac{1}{t \log ^{2}\left(\frac{1}{t}\right)}\left[1+\mathcal{O}\left(|\log t|^{-1}\right)\right], \quad \text { as } \quad t \downarrow 0,
$$

so that $\mathcal{I} \in L_{\mathrm{loc}}^{1}([0, \infty))$ and $\mathcal{I} \notin L_{\mathrm{loc}}^{p}([0, \infty))$, for every $p>1$. In addition,

$$
\mathcal{I}(t) \sim e^{t}+\mathcal{O}\left(t^{-1}\right), \quad \text { as } \quad t \rightarrow+\infty
$$

Furthermore, and above all, such a function is a Sonine kernel, namely there exists another function $\mathcal{J}(t)$ such that:

$$
\int_{0}^{t} \mathrm{~d} s \mathcal{I}(t-s) \mathcal{J}(s)=1 \quad \forall t \geq 0
$$

Precisely, $\mathcal{J}(t):=-\gamma-\log t$.

Now, we can explain why (2.4) solves (2.3), provided that $\psi_{t} \in \mathcal{D}\left(H_{\alpha, \mathbf{y}}\right)$ for every $t \geq 0$. For the sake of simplicity we assume here $q(0)=0$, since this is not restrictive (the argument if $q(0) \neq 0$ is analogous, up to 
further computations). First, we note that:

$$
\begin{aligned}
\imath \partial_{t} \psi_{t}(\mathbf{x}) & =\left(-\Delta U_{0}(t) \psi_{0}\right)(\mathbf{x})-\frac{q(t)}{2 \pi}+\frac{1}{2 \pi} \int_{0}^{t} \mathrm{~d} \tau \partial_{\tau} U_{0}(t-\tau ;|\mathbf{x}-\mathbf{y}|) q(\tau) \\
& =\left(-\Delta U_{0}(t) \psi_{0}\right)(\mathbf{x})-\frac{1}{2 \pi} \int_{0}^{t} \mathrm{~d} \tau U_{0}(t-\tau ;|\mathbf{x}-\mathbf{y}|) \dot{q}(\tau),
\end{aligned}
$$

where we used the fact that $i \partial_{t} U_{0}(t) \psi_{0}=-\Delta U_{0}(t) \psi_{0}$. Hence, applying the Fourier transform on $\mathbb{R}^{2}$, the above expression reads (setting $k=|\mathbf{k}|$ ):

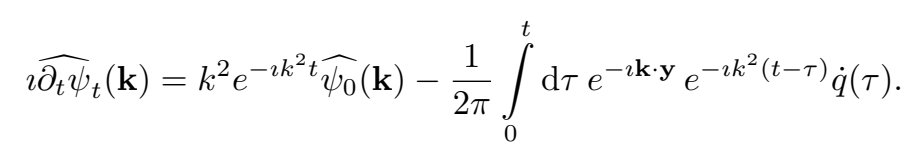

On the other hand, the Fourier transform of $H_{\alpha, \mathbf{y}} \psi_{t}$ turns out to be

$$
\begin{aligned}
k^{2}\left(\widehat{\psi}_{t}(\mathbf{k})-\frac{1}{2 \pi} \frac{q(t) e^{-\imath \mathbf{k} \cdot \mathbf{y}}}{k^{2}+\lambda}\right)-\frac{\lambda}{2 \pi} \frac{q(t) e^{-\imath \mathbf{k} \cdot \mathbf{y}}}{k^{2}+\lambda} & \\
=k^{2} e^{-\imath k^{2} t} \widehat{\psi}_{0}(\mathbf{k})+\frac{1}{2 \pi} \int_{0}^{t} \mathrm{~d} \tau e^{-\imath \mathbf{k} \cdot \mathbf{y}} \partial_{\tau}\left(e^{-\imath k^{2}(t-\tau)}\right) q(\tau)-\frac{q(t) e^{-\imath \mathbf{k} \cdot \mathbf{y}}}{2 \pi} & =k^{2} e^{-\imath k^{2} t} \widehat{\psi}_{0}(\mathbf{k})-\frac{1}{2 \pi} \int_{0}^{t} \mathrm{~d} \tau e^{-\imath \mathbf{k} \cdot \mathbf{y}} e^{-\imath k^{2}(t-\tau)} \dot{q}(\tau),
\end{aligned}
$$

which is equal to the r.h.s. of (2.5). Summing up, if $\psi_{t} \in \mathcal{D}\left(H_{\alpha, \mathbf{y}}\right)$ for every $t \geq 0$, then the ansatz (2.4) solves (2.3). In fact, some regularity for the charge $q$ is also required in order to make rigorous the previous computation. However, since in view of (2.4), the regularity of $\psi_{t}$ is due to $q(t)$, this request is somehow hidden in the assumption $\psi_{t} \in \mathcal{D}\left(H_{\alpha, \mathbf{y}}\right)$.

At this point it is evident that the central question is the behavior of $q(t)$, or in other words, the detection of the proper evolution equation for $q(t)$ (which will turn out to be a Volterra integral equation of the first kind). The argument below is just a formal derivation of the charge equation (a more rigorous way that exploits Laplace transform can be found in [47]), but is interesting since stresses the underlying link between this equation and the boundary condition present in the operator domain.

In order to guarantee that $\psi_{t} \in \mathcal{D}\left(H_{\alpha, \mathbf{y}}\right)$, the boundary condition must be satisfied, i.e.,

$$
\phi_{\lambda, t}(\mathbf{y})=\frac{1}{2 \pi} \int_{\mathbb{R}^{2}} \mathrm{~d} \mathbf{k} e^{\imath \mathbf{k} \cdot \mathbf{y}} \widehat{\phi}_{\lambda, t}(\mathbf{k})=\left(\alpha+\frac{1}{2 \pi} \log \frac{\sqrt{\lambda}}{2}-\frac{\gamma}{2 \pi}\right) q(t) .
$$

Moreover, since $\phi_{\lambda, t}=\psi_{t}-q(t) G^{\lambda}(\cdot-\mathbf{y})$,

$$
\begin{aligned}
\frac{1}{2 \pi} \int_{\mathbb{R}^{2}} \mathrm{~d} \mathbf{k} e^{\imath \mathbf{k} \cdot \mathbf{y}}\left\{e^{-\imath k^{2} t} \widehat{\psi}_{0}(\mathbf{k})+\frac{\imath}{2 \pi} \int_{0}^{t} \mathrm{~d} \tau e^{-\imath \mathbf{k} \cdot \mathbf{y}} e^{-\imath k^{2}(t-\tau)} q(\tau)-\frac{1}{2 \pi} \frac{q(t) e^{-\imath \mathbf{k} \cdot \mathbf{y}}}{k^{2}+\lambda}\right\} & =\left(\alpha+\frac{1}{2 \pi} \log \frac{\sqrt{\lambda}}{2}-\frac{\gamma}{2 \pi}\right) q(t) .
\end{aligned}
$$

Combining the last diverging term on the l.h.s. with the second one via an integration by parts, we get:

$$
\begin{aligned}
\frac{1}{2 \pi} \int_{\mathbb{R}^{2}} \mathrm{~d} \mathbf{k}\left\{e^{\imath \mathbf{k} \cdot \mathbf{y}} e^{-\imath k^{2} t} \widehat{\psi}_{0}(\mathbf{k})-\frac{1}{2 \pi\left(k^{2}+\lambda\right)} \int_{0}^{t} \mathrm{~d} \tau e^{-\imath k^{2}(t-\tau)}[\dot{q}(\tau)-\imath \lambda q(\tau)]\right\} & =\left(\alpha+\frac{1}{2 \pi} \log \frac{\sqrt{\lambda}}{2}-\frac{\gamma}{2 \pi}\right) q(t) .
\end{aligned}
$$


The integral in $\mathbf{k}$ of the second term on the 1.h.s. contains an infrared singularity for $t=\tau$ which is proportional to $\log (t-\tau)$ : in fact by [48, Eqs. 3.722.1 and 3.722.3]

$$
\begin{aligned}
\int_{\mathbb{R}^{2}} \mathrm{~d} \mathbf{k} \frac{e^{-\imath k^{2}(t-\tau)}}{k^{2}+\lambda}=-\pi e^{\imath \lambda(t-\tau)}[\operatorname{ci}(\lambda(t-\tau)) & -\imath \operatorname{si}(\lambda(t-\tau))] \\
& =-\pi e^{\imath \lambda(t-\tau)}(\gamma+\log \lambda+\log (t-\tau))+e^{\imath \lambda(t-\tau)} Q(\lambda ; t-\tau)
\end{aligned}
$$

where $\operatorname{si}(\cdot)$ and $\operatorname{ci}(\cdot)$ stand for the sine and cosine integral functions (see [49, Eqs. 5.2.1 and 5.2.2] for the definition) and, by [49, Eq. 5.2.16],

$$
Q(\lambda ; t-\tau):=-\pi\left(\sum_{n=1}^{\infty} \frac{\left(-(t-\tau)^{2} \lambda^{2}\right)^{n}}{2 n(2 n) !}-\imath \operatorname{si}((t-\tau) \lambda)\right)
$$

(note that $\left.Q(0 ; t-\tau)=-\imath \pi^{2} / 2\right)$. Hence, we obtain that:

$$
\begin{aligned}
\left(U_{0}(t) \psi_{0}\right)(\mathbf{y})-\left(\alpha+\frac{1}{2 \pi} \log \frac{\sqrt{\lambda}}{2}\right. & \left.+\frac{\gamma}{2 \pi}\right) q(t) \\
& =-\frac{1}{4 \pi} \int_{0}^{t} \mathrm{~d} \tau\left(\gamma+\log (t-\tau)+\log \lambda-\frac{1}{\pi} Q(\lambda ; t-\tau)\right) \partial_{\tau}\left(e^{\imath \lambda(t-\tau)} q(\tau)\right)
\end{aligned}
$$

and taking the formal limit $\lambda \rightarrow 0$ (notice the exact cancellation of the diverging $\log \lambda$ terms)

$$
\left(U_{0}(t) \psi_{0}\right)(\mathbf{y})-\left(\alpha-\frac{1}{2 \pi} \log 2+\frac{\gamma}{2 \pi}-\frac{\imath}{8}\right) q(t)=-\frac{1}{4 \pi} \int_{0}^{t} \mathrm{~d} \tau(\gamma+\log (t-\tau)) \dot{q}(\tau) .
$$

Finally, applying the convolution integral operator defined by $\mathcal{I}$ and using the Sonine property, suitably rearranging terms, one has:

$$
q(t)+\int_{0}^{t} \mathrm{~d} \tau \mathcal{I}(t-\tau)\left(4 \pi \alpha-2 \log 2+2 \gamma-\frac{i \pi}{2}\right) q(\tau)=f(t)
$$

where:

$$
f(t)=4 \pi \int_{0}^{t} \mathrm{~d} \tau \mathcal{I}(t-\tau)\left(U_{0}(\tau) \psi_{0}\right)(\mathbf{y})
$$

which is what is usually called charge equation.

It is clear that the previous computations are just formal. Moreover, the actual strategy to prove that (2.4) solves (2.3) is the converse of what we made. Indeed, the main steps should be the following:

(i) proving that (2.6) has a unique solution, at least on a small interval;

(ii) proving that it also displays such a regularity that $\psi_{t} \in \mathcal{D}\left(H_{\alpha, \mathbf{y}}\right)$ (and thus (2.4) satisfies (2.3));

(iii) proving that the solution of (2.6) (and consequently the solution of (2.3)) is global in time.

However, one can immediately see that this strategy is not the most suitable in the linear case, since classical theory of self-adjoint operators provides immediately (i)-(iii). In addition, point (ii) is not easy to prove in a direct way since the integral operator:

$$
(I g)(t):=\int_{0}^{t} \mathrm{~d} \tau \mathcal{I}(t-\tau) g(\tau),
$$

which is the main feature of the charge equation, has no regularizing properties in Sobolev spaces (due to its highly singular behavior at the origin) and this prevents establishment on the suitable regularity of $\phi_{\lambda, t}$. More in detail, even if $g$ is a smooth function, $I g$ may present a very rough behavior. For instance, setting $g \equiv 1$, one can see that $I g(t)=\int_{0}^{t} \mathrm{~d} \tau \mathcal{I}(\tau)=\nu(t, 0)$ is not even in $H_{\text {loc }}^{\theta}\left(\mathbb{R}^{+}\right)$, if $\theta>1 / 2$, while it belongs to $H_{\text {loc }}^{1 / 2}\left(\mathbb{R}^{+}\right)$.

On the other hand, in the nonlinear case, when the classical theory is not available, the strategy hinted before is the unique path one can follow, provided that one can manage point (ii) in spite of the singular behavior of the operator $I$. 
Remark 2.4. The lack of regularizing properties of the integral operator $I$ is the main difference between the $2 \mathrm{~d}$ case and the $1 \mathrm{~d}$ and $3 \mathrm{~d}$ ones. Indeed, in odd dimension the resulting charge equation displays the $1 / 2$-Abel kernel $\frac{C_{\beta}}{t^{1-\beta}}, \beta \in(0,1)$, as integral kernel (in place of $\mathcal{I}$ ), which has sufficient smoothing properties (see $[32,50]$ ) to overcome the regularity issues.

\section{Nonlinear Point Interactions}

As we mentioned before, the method based on the investigation of the Duhamel formula and the charge equation, although not necessary in the linear case, is the one that solely allows an easy generalization to the nonlinear problem.

Precisely, this extension is done by analogy, simply assuming that the strength of the interaction $\alpha$ depends itself on the charge in a nonlinear way (of power type), i.e.,

$$
\alpha=\beta_{0}|q(t)|^{2 \sigma}, \quad \beta_{0} \in \mathbb{R}, \sigma \in \mathbb{R}^{+} .
$$

Then, one has to follow the strategy suggested before, namely one has to prove that the function $\psi_{t}$ defined by (2.4) solves (2.3) at least in a weak sense, provided that there is a unique and sufficiently regular solution of the charge equation. It is clear that, in view of (3.1), (2.6) reads:

$$
q(t)+4 \pi \beta_{0} \int_{0}^{t} \mathrm{~d} \tau \mathcal{I}(t-\tau)|q(\tau)|^{2 \sigma} q(\tau)-2\left(\log 2-\gamma+\frac{\imath \pi}{4}\right) \int_{0}^{t} \mathrm{~d} \tau \mathcal{I}(t-\tau) q(\tau)=f(t),
$$

where $f(t)$ is given again by (2.7).

This problem has been solved in [42] by the authors. However, since an exhaustive presentation of the proof would require the management of several hard and subtle technical issues, here we just give some hints on the strategy used to overcome items (i)-(iii).

\subsection{Sketch of the strategy}

First, we want to point out that in [42] we dealt with the weak solution of (2.3), namely with a function $\psi_{t} \in V$ that satisfies:

$$
\left\{\begin{array}{l}
\imath \frac{\mathrm{d}}{\mathrm{d} t}\left\langle\chi \mid \psi_{t}\right\rangle=\mathcal{F}_{\alpha, \mathbf{y}}\left[\chi, \psi_{t}\right] \mid\left\{\alpha=\beta_{0}|q(t)|^{2 \sigma}\right\} \\
\psi_{t=0}=\psi_{0}
\end{array}\right.
$$

for any $\chi=\chi_{\lambda}+q_{\chi} G^{\lambda}(\cdot-\mathbf{y}) \in V$, where $\langle\cdot \mid \cdot\rangle$ is the inner product of $L^{2}\left(\mathbb{R}^{2}\right)$ and

$$
\begin{aligned}
\mathcal{F}_{\alpha, \mathbf{y}}\left[\chi, \psi_{t}\right]_{\mid\left\{\alpha=\beta_{0}|q(t)|^{2 \sigma}\right\}}:=\int_{\mathbb{R}^{2}} \mathrm{~d} \mathbf{x}\left\{\nabla \chi_{\lambda}^{*} \cdot \nabla \phi_{\lambda, t}+\lambda \chi_{\lambda}^{*} \phi_{\lambda, t}-\lambda \chi^{*} \psi_{t}\right\} & \\
& +\left(\beta_{0}|q(t)|^{2 \sigma}+\frac{1}{2 \pi} \log \frac{\sqrt{\lambda}}{2}+\frac{\gamma}{2 \pi}\right) q_{\chi}^{*} q(t) .
\end{aligned}
$$

The search for a strong solution seems to be out of reach at the moment. We will explain the reason below.

Remark 3.1. We stress that $\mathcal{F}_{\alpha, \mathbf{y}}[\cdot, \cdot]_{\mid\left\{\alpha=\beta_{0}|q(t)|^{2 \sigma}\right\}}$ is nothing but the nonlinear analog of the sesquilinear form associated with the quadratic form $\mathcal{F}_{\alpha, \mathbf{y}}$ defined by (2.2) and hence is the natural choice for the definition of weak solution.

Point (i) is the simplest one since it exploits some well-known results on nonlinear Volterra integral equations (see, e.g., [51,52]). Thus, one almost immediately finds that (3.2) has a unique continuous solution $q(t)$ on a maximal existence interval $\left[0, T_{*}\right)$, where $T_{*}$ is possibly infinite.

On the other hand, the central and more delicate point is (ii). In particular, one can prove that, in order to have that $\psi_{t} \in V$ and satisfies (3.3) in the maximal existence time, it is sufficient that the $q \in H^{1 / 2}(0, T)$ for all $T<T_{*}$. However, as we suggested in the previous section, this is not an easy task due to the lack or regularizing properties of the integral operator $I$, defined by the Volterra function $\mathcal{I}$. In addition, since as we showed before the action of the operator $I$ can destroy the regularity even of smooth functions, the strategy of the $1 \mathrm{~d}$ and $3 \mathrm{~d}$ cases (where we recall that the integral kernel $\mathcal{I}$ is replaced by the $1 / 2$-Abel one) namely solving smoother problems with more regular initial data and then using a density argument, is forbidden too.

Consequently, the unique available strategy, which is the one exploited in [42], is that of:

- developing a contraction argument on a possibly small interval $[0, T]$; 
- repeating the same argument on consecutive intervals with suitable modifications of (3.2);

- proving that the attachments preserve the regularity at the connection points and allow to cover any closed and bounded interval strictly contained in $\left[0, T_{*}\right)$.

Such a procedure works since the operator $I$ displays the following contractive property (see $[42,43]$ for the proof):

$$
\|I g\|_{H^{1 / 2}(0, T)} \leq C_{T}\left(\|g\|_{L^{\infty}(0, T)}+\|g\|_{H^{1 / 2}(0, T)}\right)
$$

where $C_{T} \rightarrow 0$, as $T \rightarrow 0$.

Finally, the proof of point (iii) consists of detecting sufficient conditions in order to claim that $T_{*}=+\infty$. The first step in this direction is the proof of the the conservation of the mass, i.e., $M(t):=\left\|\psi_{t}\right\|_{L^{2}\left(\mathbb{R}^{2}\right)}$, and, especially, of the energy, i.e.,

$$
E(t):=\left\|\phi_{1, t}\right\|_{H^{1}\left(\mathbb{R}^{2}\right)}^{2}+\left(\frac{\beta_{0}}{\sigma+1}|q(t)|^{2 \sigma}+\frac{\gamma-\log 2}{2 \pi}\right)|q(t)|^{2} .
$$

Hence, a classical blow-up alternative analysis shows that in the so-called defocusing case, i.e., $\beta_{0}>0$, the solution is global in time, whereas in the focusing case, i.e., $\beta_{0}<0, T_{*}$ may be both finite and infinite, depending on the initial datum $\psi_{0}$.

\subsection{Further remarks}

The methods mentioned before to manage points (ii) and (iii) have proved to be full of subtle and hard technical issues. An extensive discussion of these goes beyond the aims of this proceeding and has been done in detail in [42]. However there are points that deserve some comments.

First, we want to stress an immediate reason that makes the proof the strong version of (3.3) out of reach at the moment. This is again connected to point (ii) and, precisely, to the contracting properties of $I$. It is in fact possible to establish an analogous of (3.4) also for $H^{1}$-functions (actually for any $\nu \in(1 / 2,1]$ ), which is the regularity required to get $\psi_{t} \in \mathcal{D}\left(H_{\alpha, \mathbf{y}}\right)$ up to the proof of the boundary condition. However, in this case it is necessary to assume that $q(0)=0$, which is an unnatural assumption and, in addition, prevents the possibility of using the attachments technique highlighted in the previous section. Thus, point (ii) cannot be proved for the $H^{1}$ regularity and this prevents at the moment the possibility of finding strong solutions of (3.3).

Furthermore, both the attachments technique and the proof of the energy conservation call for a further regularity of the charge. The former issue is due to the failure of the Hardy inequality for $H^{1 / 2}$-functions (see $[36,53]$ ), that prevents the attachment of two $H^{1 / 2}$-functions on consecutive intervals to be in $H^{1 / 2}$, in general. The latter, on the contrary, is due to the integration of the derivative of the charge, which is necessary in the computations, but which have no meaning as $q$ is not absolutely continuous, in general. However, if one proves that $q$ is $\log$-Hölder continuous, namely, that its modulus of continuity is controlled by a logarithmic function (in place of a fractional power function), then both the issues can be overcome. Indeed, in this case the attachments can be proved to be licit and one can develop a duality pairing argument in order to bypass the problem on integrating $\dot{q}$.

Unfortunately, the proof of such a further regularity for the charge requires some extra-assumptions on the initial datum $\psi_{0}$. Precisely, its regular part $\phi_{\lambda, 0}$ has to satisfy:

$$
(1+k)^{\varepsilon} \widehat{\phi}_{\lambda, 0} \in L^{1}\left(\mathbb{R}^{2}\right), \quad \text { for some } \varepsilon>0,
$$

which is a restriction with respect to the natural assumptions that only state $\psi_{0} \in V$.

Finally, it is worth recalling that the contractive argument needed to manage point (ii) requests a slightly restrictive assumption on the power of the nonlinearity. Precisely, one must suppose that $\sigma \geq 1 / 2$. This is due to the fact that otherwise one cannot prove Lipschitz continuity of the map $g \mapsto|g|^{2 \sigma} g$ between $H^{1 / 2}(0, T) \cap L^{\infty}(0, T)$ and itself, with a constant that do not blow up as $T \rightarrow 0$; namely, with a constant that do not compensate the good contractive properties of $I$.

\section{References}

[1] Cacciapuoti C., Carlone R., Figari R. A solvable model of a tracking chamber. Rep. Math. Phys., 2007, 59(3), P. $337-349$.

[2] Carlone R., Figari R., Negulescu C. A model of a quantum particle in a quantum environment: a numerical study. Commun. Comput. Phys., 2015, 18(1), P. 247-262.

[3] Carlone R., Figari R., Negulescu C. The quantum beating and its numerical simulation. J. Math. Anal. Appl., 2017, 450(2), P. 1294-1316.

[4] Figari R., Teta A. From quantum to classical world: emergence of trajectories in a quantum system. Math. Mech. Complex Syst., 2016, 4(3-4), P. 235-254.

[5] Cacciapuoti C., Figari R., Posilicano A. Effective equation for a system of mechanical oscillators in an acoustic field. Asymptot. Anal., 2015, 91(3-4), P. 253-264. 
[6] Cacciapuoti C., Fermi D., Posilicano A. Relative-zeta and Casimir energy for a semitransparent hyperplane selecting transverse modes. Advances in quantum mechanics, P. 71-97, Springer INdAM Ser., 18, Springer, Cham, 2017.

[7] Cacciapuoti C., Carlone R., Figari R. Perturbations of eigenvalues embedded at threshold: two-dimensional solvable models. J. Math. Phys., 2011, 52(8), 083515, 12 pp.

[8] Albeverio S., Gesztesy F., Høegh-Krohn R., Holden H. Solvable Models in Quantum Mechanics. Texts and Monographs in Physics, Springer-Verlag, New York, 1988.

[9] Cacciapuoti C., Carlone R., Noja D., Posilicano A. The one-dimensional Dirac equation with concentrated nonlinearity. SIAM J. Math. Anal., 2017, 49(3), P. 2246-2268.

[10] Carlone R., Malamud M., Posilicano A. On the spectral theory of Gesztesy-Šeba realizations of 1-D Dirac operators with point interactions on a discrete set. J. Differential Equations, 2013, 254(9), P. 3835-3902.

[11] Adami R., Cacciapuoti C., Finco D., Noja D. Fast solitons on star graphs. Rev. Math. Phys., 2011, 23(4), P. 409-451.

[12] Adami R., Cacciapuoti C., Finco D., Noja D. On the structure of critical energy levels for the cubic focusing NLS on star graphs. J. Phys. $A, 2012,45(19), 192001,7 \mathrm{pp}$.

[13] Adami R., Cacciapuoti C., Finco D., Noja D. Constrained energy minimization and orbital stability for the NLS equation on a star graph. Ann. Inst. H. Poincaré Anal. Non Linéaire, 2014, 31(6), P. 1289-1310.

[14] Adami R., Cacciapuoti C., Finco D., Noja D. Variational properties and orbital stability of standing waves for NLS equation on a star graph. J. Differential Equations, 2014, 257(10), P. 3738-3777.

[15] Adami R., Serra E., Tilli P. Lack of ground state for NLSE on bridge-type graphs. Mathematical technology of networks, 1-11. Springer Proc. Math. Stat., 128. Springer, Cham, 2015.

[16] Adami R., Serra E., Tilli P. NLS ground states on graphs. Calc. Var. Partial Differential Equations, 2015, 54(1), P. 743-761.

[17] Adami R., Serra E., Tilli P. Threshold phenomena and existence results for NLS ground states on graphs. J. Funct. Anal., 2016, 271(1), P. 201-223.

[18] Adami R., Serra E., Tilli P. Negative energy ground states for the $L^{2}$-critical NLSE on metric graphs. Comm. Math. Phys., 2017, 352(1), P. $387-406$.

[19] Berkolaiko G., Kuchment P. Introduction to quantum graphs, Mathematical Surveys and Monographs 186, AMS, Providence, RI, 2013.

[20] Cacciapuoti C., Finco D., Noja D. Topology-induced bifurcations for the nonlinear Schrödinger equation on the tadpole graph. Phys. Rev. $E$ (3), 2015, 91(1), article number 013206, 8 pp.

[21] Gnutzmann S., Smilansky U., Derevyanko S. Stationary scattering from a nonlinear network. Phys. Rev. A, 2011, 83(3), article number 033831, $6 \mathrm{pp}$.

[22] Noja D. Nonlinear Schrödinger equation on graphs: recent results and open problems. Philos. Trans. R. Soc. Lond. Ser. A Math. Phys. Eng. Sci., 2014, 372(2007), 20130002, 20 pp.

[23] Noja D., Pelinovsky D., Shaikhova G. Bifurcations and stability of standing waves in the nonlinear Schrödinger equation on the tadpole graph. Nonlinearity, 2015, 28(7), P. 2343-2378.

[24] Serra E., Tentarelli L. Bound states of the NLS equation on metric graphs with localized nonlinearities. J. Differential Equations, 2016, 260(7), P. 5627-5644

[25] Serra E., Tentarelli L. On the lack of bound states for certain NLS equations on metric graphs. Nonlinear Anal., 2016, 145, P. 68-82.

[26] Tentarelli L. NLS ground states on metric graphs with localized nonlinearities. J. Math. Anal. Appl., 2016, 433(1), P. 291-304.

[27] Carlone R., Exner P. Dynamics of an electron confined to a "hybrid plane" and interacting with a magnetic field. Rep. Math. Phys., 2011, 67(2), P. 211-227.

[28] Carlone R., Posilicano A. A quantum hybrid with a thin antenna at the vertex of a wedge. Phys. Lett. A, 2017, 381(12), P. 1076-1080.

[29] Exner P., Šeba P. Quantum motion on a half-line connected to a plane. J. Math. Phys., 1987, 28(2), P. 386-391.

[30] Exner P., Šeba P. Resonance statistics in a microwave cavity with a thin antenna. Phys. Lett. A, 1997, 228(3), P. 146-150.

[31] Exner P., Šeba P. A "hybrid plane" with spin-orbit interaction. Russ. J. Math. Phys., 2007, 14(4), P. $430-434$.

[32] Adami R., Teta A. A Class of Nonlinear Schrödinger Equations with Concentrated Nonlinearity. J. Funct. Anal., 2001, 180(1), P. 148-175.

[33] Adami R., Dell'Antonio G., Figari R., Teta A. The Cauchy problem for the Schrödinger equation in dimension three with concentrated nonlinearity. Ann. Inst. H. Poincaré Anal. Non Linéaire, 2003, 20(3), P. 477-500.

[34] Adami R., Dell'Antonio G., Figari R., Teta A. Blow-up solutions for the Schrödinger equation in dimension three with a concentrated nonlinearity. Ann. Inst. H. Poincaré Anal. Non Linéaire, 2004, 21(1), P. 121-137.

[35] Cacciapuoti C., Finco D., Noja D., Teta A. The NLS equation in dimension one with spatially concentrated nonlinearities: the pointlike limit. Lett. Math. Phys., 2014, 104(12), P. 1557-1570.

[36] Cacciapuoti C., Finco D., Noja D., Teta A. The point-like limit for a NLS equation with concentrated nonlinearity in dimension three. $J$. Funct. Anal., 2017, 273(5), P. 1762-1809.

[37] M. Correggi, G. Dell'Antonio, Decay of a Bound State under a Time-Periodic Perturbation: A Toy Case. J. Phys. A: Math. Gen., 2005, 38, P. 4769-4781.

[38] Correggi M., Dell'Antonio G., Figari R., Mantile A. Ionization for Three Dimensional Time-Dependent Point Interactions. Comm. Math. Phys., 2005, 257(1), P. 169-192.

[39] Costin O., Costin R.D., Lebowitz J.L., Rokhlenko A. Evolution of a model quantum system under time periodic forcing: conditions for complete ionization. Comm. Math. Phys., 2001, 221(1), P. 1-26.

[40] Sayapova M.R., Yafaev D.R. The evolution operator for time-dependent potentials of zero radius. Trudy Mat. Inst. Steklov, 1983, 159, P. 167-174.

[41] Carlone R., Correggi M., Figari R. Two-dimensional time-dependent point interactions. Functional Analysis and Operator Theory for Quantum Physics, 189-211, Eur. Math. Soc., Zürich, 2017.

[42] Carlone R., Correggi M., Tentarelli L. Well-posedness of the Two-dimensional Nonlinear Schrödinger Equation with Concentrated Nonlinearity, preprint arXiv:1702.03651 [math-ph], (2017).

[43] Carlone R., Fiorenza A., Tentarelli L. The action of Volterra integral operators with highly singular kernels on Hölder continuous, Lebesgue and Sobolev functions. J. Funct. Anal., 2017, 273(3), P. 1258-1294.

[44] Albeverio S., Brzezniak Z., Dabrowski L. Time-dependent propagator with point interaction. J. Phys. A, 1994, 27(14), P. 4933-4943. 
[45] Samko S.G., Kilbas A.A., Marichev O.I. Fractional integrals and derivatives. Theory and applications. Gordon and Breach Science Publishers, Yverdon, 1993.

[46] Erdélyi A., Magnus W., Oberhettinger F., Tricomi F.G. Higher transcendental functions. Vol. III, Robert E. Krieger Publishing Co., Melbourne, Fla., 1981.

[47] Adami R. A Class of Schrödinger Equations with Concentrated Nonlinearity. Ph.D. Thesis, Università degli Studi di Roma "La Sapienza", 2000.

[48] Gradshteyn I.S., Ryzhik I.M. Tables of Integrals, Series and Products. Elsevier/Academic Press, Amsterdam, 2007.

[49] Abramovitz M., Stegun I.A. Handbook of Mathematical Functions: with Formulas, Graphs, and Mathematical Tables. National Bureau of Standards Applied Mathematics Series 55, Washington D.C., 1964.

[50] Gorenflo R., Vessella S. Abel integral equations, Lecture Notes in Mathematics, vol. 1461, Springer-Verlag, Berlin, 1991, Analysis and applications.

[51] Miller R.K. Nonlinear Volterra Integral Equations. Mathematics Lecture Note Series, W.A. Benjamin Inc., Menlo Park, Calif., 1971.

[52] Miller R.K., Sell G.R. Existence, uniqueness and continuity of solutions of integral equations. Ann. Mat. Pura Appl. (4), 1968, 80, P. $135-152$.

[53] Kufner A., Persson L.E. Weighted inequalities of Hardy type. World Scientific Publishing Co., River Edge, NJ, 2003. 